\title{
Conditions for coherence transformations under incoherent operations
}

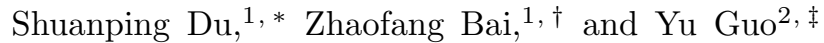 \\ ${ }^{1}$ School of Mathematical Sciences, Xiamen University, Xiamen, Fujian, 361000, China \\ ${ }^{2}$ School of Mathematics and Computer Science, Shanxi Datong University, Datong 037009, China
}

\begin{abstract}
We build the counterpart of the celebrated Nielsen's theorem for coherence manipulation in this paper. This offers an affirmative answer to the open question: whether, given two states $\rho$ and $\sigma$, either $\rho$ can be transformed into $\sigma$ or vice versa under incoherent operations [Phys. Rev. Lett. 113, 140401(2014)]. As a consequence, we find that there exist essentially different types of coherence. Moreover, incoherent operations can be enhanced in the presence of certain coherent states. These extra states are coherent catalysts: they allow uncertain incoherent operations to be realized, without being consumed in any way. Our main result also sheds a new light on the construction of coherence measures.
\end{abstract}

PACS numbers: 03.65.Ud, 03.67.-a, 03.65.Ta.

Introduction. - Superposition is a critical property of quantum system resulting in quantum coherence and quantum entanglement. Quantum coherence and also entanglement provide the important resource for quantum information processing, for example, Deutschs algorithm, Shors algorithm, teleportation, superdense coding and quantum cryptography [1]. As with any such resource, there arises naturally the question of how it can be quantified and manipulated. Attempts have been made to find meaningful measures of entanglement [2 6 ], and also to uncover the fundamental laws of its behavior under local quantum operations and classical communication (LOCC) [2 12]. The celebrated Nielsen's theorem finds possible entanglement manipulation between bipartite entanglement states by LOCC 7]. Let $|\psi\rangle=\sum_{i=1}^{d} \sqrt{\psi_{j}}|j j\rangle$ and $|\phi\rangle=\sum_{i=1}^{d} \sqrt{\phi_{j}}|j j\rangle$ be two bipartite states whose Schmidt coefficients are ordered in decreasing order, $\psi_{1} \geq \psi_{2} \geq \cdots \geq \psi_{d}, \phi_{1} \geq \phi_{2} \geq$ $\cdots \geq \phi_{d}$. Then $|\psi\rangle \rightarrow|\phi\rangle$ by LOCC if and only if $\left(\psi_{1}, \psi_{2}, \cdots, \psi_{d}\right) \prec\left(\phi_{1}, \phi_{2}, \cdots, \phi_{d}\right)$. This reveals a partial ordering on the entangled states and connects quantum entanglement to the algebraic theory of majorization.

In [13], the researchers establish a rigorous framework for the quantification of coherence as a resource following the viewpoints that have been established for entanglement in [6]. And the setting of single copies of coherent states is of considerable interest from the practical point of view as this is most readily accessible in the laboratory. It is expected that theory of coherence manipulation can be established that proceeds along analogous developments in entanglement theory [13]. The aim of this paper is to build the counterpart of the Nielsen's theorem for coherence manipulation. What is amazing is that majorization is also the key ingredient. It provides

\footnotetext{
*Electronic address: shuanpingdu@yahoo.com

${ }^{\dagger}$ Corresponding author; Electronic address: baizhaofang@xmu.edu.cn

¥Electronic address: guoyu3@aliyun.com
}

the relevant structure that determines the interconvertibility of coherent states.

Majorization is an active research area in linear algebra. We use Chap. 2 of [14] as our principal reference on majorization. Suppose $x=\left(x_{1}, x_{2}, \cdots, x_{d}\right)^{t}$ and $y=\left(y_{1}, y_{2}, \cdots, y_{d}\right)^{t}$ are real d-dimensional vectors, here $x=\left(x_{1}, x_{2}, \cdots, x_{d}\right)^{t}$ denotes the transpose of row vector $\left(x_{1}, x_{2}, \cdots, x_{d}\right)$. Then $x$ is majorized by $y$ (equivalently $y$ majorizes $x$ ), written $x \prec y$, if for each $k$ in the range $1, \cdots, d, \sum_{i=1}^{k} x_{i}^{\downarrow} \leq \sum_{i=1}^{k} y_{i}^{\downarrow}$ with equality holding when $k=d$, and where the $x_{i}^{\downarrow}$ indicates that elements are to be taken in descending order, so, for example, $x_{1}^{\downarrow}$ is the largest element in $\left(x_{1}, \cdots, x_{d}\right)$. The majorization relation is a partial order on real vectors, with $x \prec y$ and $y \prec x$ if and only if $x^{\downarrow}=y^{\downarrow}$.

In the following, we introduce the concepts of incoherent states and incoherent operations which are from [13]. Let $\mathcal{H}$ be a finite dimensional Hilbert space with $\operatorname{dim}(\mathcal{H})=d$. Fixing a particular basis $\{|i\rangle\}_{i=1}^{d}$, we call all density operators (quantum states) that are diagonal in this basis incoherent, and this set of quantum states will be labelled by $\mathcal{I}$, all density operators $\rho \in \mathcal{I}$ are of the form

$$
\rho=\sum_{i=1}^{d} \lambda_{i}|i\rangle\langle i| .
$$

Quantum operations are specified by a finite set of Kraus operators $\left\{K_{n}\right\}$ satisfying $\sum_{n} K_{n}^{\dagger} K_{n}=I, I$ is the identity operator on $\mathcal{H}$. Quantum operations are incoherent if they fulfil $K_{n} \rho K_{n}^{\dagger} / \operatorname{Tr}\left(K_{n} \rho K_{n}^{\dagger}\right) \in \mathcal{I}$ for all $\rho \in \mathcal{I}$ and for all $n$.

Results. - To state our central result linking coherence manipulation with majorization, we need some notation. Suppose $|\psi\rangle=\sum_{i=1}^{d} \psi_{i}|i\rangle$ and $|\phi\rangle=\sum_{i=1}^{d} \phi_{i}|i\rangle$ are any pure states. $|\psi\rangle \stackrel{I C O}{\longrightarrow}|\phi\rangle$, read " $|\psi\rangle$ transforms incoherently to $|\phi\rangle$ " indicates that $|\psi\rangle\langle\psi|$ transforms to $|\phi\rangle\langle\phi|$ by incoherent operations. Then we have the following:

Theorem 1. $|\psi\rangle$ transforms to $|\phi\rangle$ using incoherent operations if and only if $\left(\left|\psi_{1}\right|^{2}, \cdots,\left|\psi_{d}\right|^{2}\right)^{t}$ is majorized 
by $\left(\left|\phi_{1}\right|^{2}, \cdots,\left|\phi_{d}\right|^{2}\right)^{t}$. More succinctly,

$$
\begin{aligned}
& |\psi\rangle \stackrel{I C O}{\longrightarrow}|\phi\rangle \text { iff } \\
& \left(\left|\psi_{1}\right|^{2}, \cdots,\left|\psi_{d}\right|^{2}\right)^{t} \prec\left(\left|\phi_{1}\right|^{2}, \cdots,\left|\phi_{d}\right|^{2}\right)^{t} .
\end{aligned}
$$

One direct consequence of Theorem 1 is that there exist pairs $|\psi\rangle$ and $|\phi\rangle$ with neither $|\psi\rangle \stackrel{I C O}{\longrightarrow}|\phi\rangle$ nor $|\phi\rangle \stackrel{I C O}{\longrightarrow}$ $|\psi\rangle$. For example, $d=3$,

$$
\begin{aligned}
& |\psi\rangle=\sqrt{0.4}|1\rangle+\sqrt{0.3}|2\rangle+\sqrt{0.3}|3\rangle, \\
& |\phi\rangle=\sqrt{0.5}|1\rangle+\sqrt{0.1}|2\rangle+\sqrt{0.4}|3\rangle .
\end{aligned}
$$

These provide an example of essentially different types of coherence, from the point of view of incoherent operations. We will say that $|\psi\rangle$ and $|\phi\rangle$ are incomparable in coherence. In addition, for any two pure states $|\psi\rangle,|\phi\rangle$, $|\psi\rangle$ and $|\phi\rangle$ can be incomparable with respect to incoherence under a change of basis. This may seem odd at first, but it turns out that coherence is a basis dependent phenomenon.

For entanglement transformations, a major interest has been the catalysis. This enables the conversion between two initially inconvertible entangled states assisted by a lent entangled state, which is recovered at the end of the process [10, 15-19]. For two states $|\psi\rangle$ and $|\phi\rangle$ which are incomparable in coherence, if $|\psi\rangle|\delta\rangle \stackrel{I C O}{\longrightarrow}|\phi\rangle|\delta\rangle$, we say $|\psi\rangle$ is transformed into $|\phi\rangle$ under coherence-assisted incoherent operation, and $|\delta\rangle$ is called a coherent catalyst. This state acts much like a catalyst in a chemical reaction: its presence allows a previously forbidden transformation to be realized, and since it is not consumed it can be reused. Here we use the phrase "coherence-assisted" because $|\delta\rangle$ must be coherent. Combining Theorem 1 and proofs of Lemma 1, Lemma 2 and Lemma 3 in [10], we immediately have the following interesting results:

(i) No incoherent transformation can be catalyzed by a maximally coherent state $\left|\psi_{d}\right\rangle=\sum_{k=1}^{d} \frac{1}{\sqrt{d}}|k\rangle$. This shows a surprising property of coherent catalysts: they must be partially coherent. If the catalyst has not enough coherence, then $|\psi\rangle$ can not be transformed into $|\phi\rangle$ with certainty, but if it has too much then the result is same.

(ii) Two states are interconvertible (i.e., both $|\psi\rangle \rightarrow$ $|\phi\rangle$ and $|\phi\rangle \rightarrow|\psi\rangle)$ under coherence-assisted incoherent operation if and only if they are equivalent up to a permutation of diagonal unitary transformations. One consequence of this result is that if a transition that is forbidden under incoherent operation can be catalyzed (i.e. $|\psi\rangle \nrightarrow|\phi\rangle$ under incoherent operation but $|\psi\rangle|\delta\rangle \rightarrow|\phi\rangle|\delta\rangle$ for some $|\delta\rangle$ ), then the reverse transition (from $|\phi\rangle \rightarrow|\psi\rangle$ can not be catalyzed. In particular, only transitions between incomparable states may be catalyzed.

(iii) $|\psi\rangle \rightarrow|\phi\rangle$ under coherence-assisted incoherent operation only if both $\left|\psi_{1}\right| \leq\left|\phi_{1}\right|$ and $\left|\psi_{d}\right| \geq\left|\phi_{d}\right|$.

Theorem 1 provides a necessary condition for coherence measures. By [13], coherence measures should satisfy the monotonicity under incoherent operations, i.e.,
$\mathcal{C}(\Phi(\rho)) \leq \mathcal{C}(\rho)$ for any incoherent operation $\Phi$ and state $\rho$. Let $|\psi\rangle=\psi_{1}|1\rangle+\cdots+\psi_{d}|d\rangle,|\phi\rangle=\phi_{1}|1\rangle+\cdots+\phi_{d}|d\rangle$, with $\left(\left|\psi_{1}\right|^{2}, \cdots,\left|\psi_{d}\right|^{2}\right)^{t} \prec\left(\left|\phi_{1}\right|^{2}, \cdots,\left|\phi_{d}\right|^{2}\right)^{t}$. By Theorem 1 , we have $\mathcal{C}(|\phi\rangle\langle\phi|) \leq \mathcal{C}(|\psi\rangle\langle\psi|)$. This necessary condition of coherence measure implies the Result 1 in 20] is not true. That is, Wigner-Yanase-Dyson skew information

$$
\mathcal{C}(\rho, K)=-\frac{1}{2} \operatorname{Tr}\left([\sqrt{\rho}, K]^{2}\right)
$$

is not a good coherence measure since it violates this necessary condition. Assume $d=3$, let

$$
\begin{aligned}
& K=|1\rangle\langle 1|+10| 2\rangle\langle 2|+5| 3\rangle\langle 3|, \\
& |\psi\rangle=\frac{1}{\sqrt{3}}|1\rangle+\frac{1}{\sqrt{3}}|2\rangle+\frac{1}{\sqrt{3}}|3\rangle, \\
& |\phi\rangle=\frac{1}{\sqrt{2}}|1\rangle+\frac{1}{\sqrt{2}}|2\rangle .
\end{aligned}
$$

It is easy to check that $\left(\frac{1}{3}, \frac{1}{3}, \frac{1}{3}\right)^{t} \prec\left(\frac{1}{2}, \frac{1}{2}, 0\right)^{t}$ and

$$
\mathcal{C}(|\phi\rangle\langle\phi|, K)=\frac{81}{4}>\mathcal{C}(|\psi\rangle\langle\psi|, K)=\frac{122}{9} .
$$

The following construction of coherent measures is originated from Theorem 1. For arbitrary pure state $|\psi\rangle=\sum_{i=1}^{d} \psi_{i}|i\rangle$, we define $C_{l}(|\psi\rangle\langle\psi|)=\sum_{i=l}^{d}\left|\psi_{i}\right|^{2 \downarrow}$ $(l=2,3, \cdots, d)$, here $\left(\left|\psi_{1}\right|^{2 \downarrow},\left|\psi_{2}\right|^{2 \downarrow}, \cdots,\left|\psi_{d}\right|^{2 \downarrow}\right)^{t}$ is the vector obtained by rearranging the coordinates of $\left(\left|\psi_{1}\right|^{2},\left|\psi_{2}\right|^{2}, \cdots,\left|\psi_{d}\right|^{2}\right)^{t}$ in the decreasing order, and extending it over the whole set of density matrices as $C_{l}(\rho)=\min _{p_{j}, \rho_{j}} \sum_{j} p_{j} C_{l}\left(\rho_{j}\right)$, where the minimization is to be performed over all the pure-state ensembles of $\rho$, i.e., $\rho=\sum_{j} p_{j} \rho_{j}$. In [21], we show that $C_{l}$ are coherence measures.

Theorem 1 also pave the way for the following question: suppose there is a pure coherent state $|\psi\rangle=\sum_{i=1}^{d} \psi_{i}|i\rangle$ and we would like to convert it into another pure coherent state $|\phi\rangle=\sum_{i=1}^{d} \phi_{i}|i\rangle$ by incoherent operations. Which is the greatest probability of success in such a conversion? In [21], we give the explicit formula of the greatest probability $P(|\psi\rangle \stackrel{I C O}{\longrightarrow}|\phi\rangle)$. A parallel result in entanglement theory is optimal local conversion strategy between any two pure entangled states of a bipartite system [8].

Proofs. - Now we do some preparatory work to prove Theorem 1 by collecting some useful facts:

(i) For real vectors $x, y, x \prec y$ if and only if $x=A y$ for some doubly stochastic matrix. Recall that a $d \times d$ matrix $A=\left(a_{i j}\right)$ is called doubly stochastic if $a_{i j} \geq 0$ and $\sum_{i=1}^{d} a_{i j}=\sum_{j=1}^{d} a_{i j}=1$.

(ii) For every doubly stochastic matrix $A$, it is a matrix that may be written as a product of at most $d-1$ $T$-transforms. A $T$ - transform, by definition, acts as the identity on all but two matrix components. On those two components, it has the form

$$
T=\left(\begin{array}{cc}
t & 1-t \\
1-t & t
\end{array}\right),
$$

where $0 \leq t \leq 1$. In terms of transformation, $T\left(x_{1}, x_{2}, \cdots, x_{d}\right)^{t}=\left(x_{1}, \cdots, x_{i-1}, t x_{i}+(1-\right.$ 
$\left.t) x_{j}, x_{i+1}, \cdots x_{j-1},(1-t) x_{i}+t x_{j}, x_{j+1}, \cdots, x_{d}\right)^{t}$ for some indies $i, j$ and $0 \leq t \leq 1$.

(iii) Let $\pi$ be a permutation of $\{1,2, \cdots, d\}$ and $P_{\pi}$ be the permutation matrix corresponding to $\pi$ which is obtained by permuting the rows of a $d \times d$ identity matrix according to $\pi$. A permutation matrix has exactly one entry 1 in each row and each column and 0 elsewhere.

(iv) For quantum operation $\Phi(\cdot)=\sum_{n} K_{n} \cdot K_{n}^{\dagger}$, it is easy to see that $\Phi$ is incoherent if and only if every column of $K_{n}$ in the fixed basis $\{|i\rangle\}_{i=1}^{d}$ is with at most 1 nonzero entry.

Now, we are in the position to give the proof of Theorem 1.

Proof: Firstly, we can suppose all $\psi_{k}, \phi_{k}(k=$ $1,2, \cdots, d)$ are nonnegative and sorted in descending order. Indeed, in general case, let $\psi_{k}=\left|\psi_{k}\right| e^{i \alpha_{k}}$, $\phi_{k}=\left|\phi_{k}\right| e^{i \beta_{k}}$ and $\left|\psi_{\pi(1)}\right| \geq\left|\psi_{\pi(2)}\right| \geq \cdots \geq$ $\left|\psi_{\pi(d)}\right|,\left|\phi_{\sigma(1)}\right| \geq\left|\phi_{\sigma(2)}\right| \geq \cdots \geq\left|\phi_{\sigma(d)}\right|$, where $\pi, \sigma$ are two permutations of $\{1,2, \cdots, d\}$. One can define $U=P_{\pi} \operatorname{diag}\left(e^{-i \alpha_{1}}, e^{-i \alpha_{2}}, \cdots, e^{-i \alpha_{d}}\right)$ and $V=$ $P_{\sigma} \operatorname{diag}\left(e^{-i \beta_{1}}, e^{-i \beta_{2}}, \cdots, e^{-i \beta_{d}}\right)$, here $P_{\pi}$ and $P_{\sigma}$ are permutation matrices corresponding to $\pi$ and $\sigma$, respectively. Note that $U|\psi\rangle \stackrel{I C O}{\longrightarrow} V|\phi\rangle \Leftrightarrow|\psi\rangle \stackrel{I C O}{\longrightarrow}|\phi\rangle$, we can replace $|\psi\rangle$ and $|\phi\rangle$ by $U|\psi\rangle$ and $V|\phi\rangle$.

Now, we prove the "if" part. Assume that $\left(\left|\psi_{1}\right|^{2}, \cdots,\left|\psi_{d}\right|^{2}\right)^{t} \prec\left(\left|\phi_{1}\right|^{2}, \cdots,\left|\phi_{d}\right|^{2}\right)^{t}$. We will apply the inductive method.

Assume $\operatorname{dim} H=2$. If $\psi_{2}=0$, from the majorization, it follows that $\phi_{2}=0$. That is $|\psi\rangle=|\phi\rangle=|1\rangle$. Then the identity operation is the desired. Now we may suppose $\psi_{2} \neq 0$. Let $A=\left(\begin{array}{cc}a & 1-a \\ 1-a & a\end{array}\right)(0 \leq a \leq 1)$ be the doubly stochastic matrix such that

$$
\left(\begin{array}{l}
\psi_{1}^{2} \\
\psi_{2}^{2}
\end{array}\right)=\left(\begin{array}{cc}
a & 1-a \\
1-a & a
\end{array}\right)\left(\begin{array}{l}
\phi_{1}^{2} \\
\phi_{2}^{2}
\end{array}\right) .
$$

Define

$$
\begin{gathered}
K_{1}=\left(\begin{array}{cc}
\sqrt{a} \frac{\phi_{1}}{\psi_{1}} & 0 \\
0 & \sqrt{a} \frac{\phi_{2}}{\psi_{2}}
\end{array}\right), \\
K_{2}=\left(\begin{array}{cc}
0 & \sqrt{1-a} \frac{\phi_{1}}{\psi_{2}} \\
\sqrt{1-a} \frac{\phi_{2}}{\psi_{1}} & 0
\end{array}\right) .
\end{gathered}
$$

One can check that the incoherent operation whose Kraus operators are $K_{1}, K_{2}$ is the desired.

Assume the result holds true for $\operatorname{dim} H \leq d-1$, we will prove that the result holds true for $\operatorname{dim} H=d$ and divide the proof into two cases.

Case 1. There is a $k(1<k<d)$ such that $\psi_{k} \neq 0$ and $\psi_{k+1}=\cdots=\psi_{d}=0$. From the majorization, it follows that $\phi_{k+1}=\cdots=\phi_{d}=0$. The $k$ level vector $\left(\left|\psi_{1}\right|^{2}, \cdots,\left|\psi_{k}\right|^{2}\right)^{t}$ is majorized by $\left(\left|\phi_{1}\right|^{2}, \cdots,\left|\phi_{k}\right|^{2}\right)^{t}$. From the inductive assumption, there is an incoherent operation $\widetilde{\Phi}$ on $M_{k}$ (the set of all $k \times k$ level matrices) specified the Kraus operators $\widetilde{K_{n}}(n=1,2, \cdots, N)$ such that $\sum_{i=1}^{k} \psi_{i}|i\rangle \stackrel{\widetilde{\Phi}}{\rightarrow} \sum_{i=1}^{k} \phi_{i}|i\rangle$. Define $K_{n}=\widetilde{K_{n}} \oplus \frac{1}{\sqrt{N}} I_{d-k}$, then $\Phi(\cdot)=\sum_{i=1}^{N} K_{n} \cdot K_{n}^{\dagger}$ is an incoherent operation which transforms $|\psi\rangle\langle\psi|$ to $|\phi\rangle\langle\phi|$.

Case 2. $\psi_{d} \neq 0$. Let $A$ be a doubly stochastic matrix with $\left(\left|\psi_{1}\right|^{2}, \cdots,\left|\psi_{d}\right|^{2}\right)^{t}=A\left(\left|\phi_{1}\right|^{2}, \cdots,\left|\phi_{d}\right|^{2}\right)^{t}$. Note that the composition of incoherent operations are also incoherent, by the fact (ii), $A$ can be reduced to a $T$-transform for some indices $i, j$ and $0 \leq t \leq 1$. Let $\pi=(1,2, \cdots, i-1, j, i+1, \cdots, j-1, i, j+1, \cdots, d)$ be a permutation of $\{1,2, \cdots, d\}$, and

$$
\begin{gathered}
K_{1}=\sqrt{t} \operatorname{diag}\left(\frac{\phi_{1}}{\psi_{1}}, \cdots, \frac{\phi_{d}}{\psi_{d}}\right), \\
K_{2}=\sqrt{1-t} \operatorname{diag}\left(\frac{\phi_{1}}{\psi_{1}}, \cdots, \frac{\phi_{i-1}}{\psi_{i-1}}, \frac{\phi_{i}}{\psi_{j}}, \frac{\phi_{i+1}}{\psi_{i+1}}, \cdots,\right. \\
\left.\frac{\phi_{j-1}}{\psi_{j-1}}, \frac{\phi_{j}}{\psi_{i}}, \frac{\phi_{j+1}}{\psi_{j+1}}, \cdots, \frac{\phi_{d}}{\psi_{d}}\right) P_{\pi} .
\end{gathered}
$$

Then

$$
\begin{gathered}
K_{1}^{\dagger} K_{1}=t \operatorname{diag}\left(\frac{\phi_{1}^{2}}{\psi_{1}^{2}}, \cdots, \frac{\phi_{d}^{2}}{\psi_{d}^{2}}\right), \\
K_{2}^{\dagger} K_{2}=(1-t) \operatorname{diag}\left(\frac{\phi_{1}^{2}}{\psi_{1}^{2}}, \cdots, \frac{\phi_{i-1}^{2}}{\psi_{i-1}^{2}}, \frac{\phi_{j}^{2}}{\psi_{i}^{2}}, \frac{\phi_{i+1}^{2}}{\psi_{i+1}^{2}}, \cdots,\right. \\
\left.\frac{\phi_{j-1}^{2}}{\psi_{j-1}^{2}}, \frac{\phi_{i}^{2}}{\psi_{j}^{2}}, \frac{\phi_{j+1}^{2}}{\psi_{j+1}^{2}}, \cdots, \frac{\phi_{d}^{2}}{\psi_{d}^{2}}\right) .
\end{gathered}
$$

From $\left(\left|\psi_{1}\right|^{2}, \cdots,\left|\psi_{d}\right|^{2}\right)^{t}=A\left(\left|\phi_{1}\right|^{2}, \cdots,\left|\phi_{d}\right|^{2}\right)^{t}$, it follows that $K_{1}^{\dagger} K_{1}+K_{2}^{\dagger} K_{2}=I$. Furthermore, it is easy to check that $\Phi(\cdot)=\sum_{n=1}^{2} K_{n} \cdot K_{n}^{\dagger}$ transforms $|\psi\rangle\langle\psi|$ to $|\phi\rangle\langle\phi|$. Note that each column of $K_{n}(n=1,2)$ has at most one nonzero entry, so $\Phi$ is incoherent. This finishes the proof of the "if" part.

To prove the converse, we only consider the three dimensional case, other cases can be treated similarly. Now, we suppose $\operatorname{dim} H=3$ and there is an incoherent operation $\Phi$ transforms $|\psi\rangle\langle\psi|$ to $|\phi\rangle\langle\phi|$. Let

$$
\Phi(|\psi\rangle\langle\psi|)=\sum_{n} K_{n}|\psi\rangle\left\langle\psi\left|K_{n}^{\dagger}=\right| \phi\right\rangle\langle\phi| .
$$

Hence there exist complex numbers $\alpha_{n}$ such that $K_{n}|\psi\rangle=\alpha_{n}|\phi\rangle$. Let $k_{j}^{(n)}(j=1,2,3)$ be the nonzero element of $K_{n}$ at $j-t h$ column (if there is no nonzero element in $j-t h$ column, then $\left.k_{j}^{(n)}=0\right)$. Suppose $k_{j}^{(n)}$ locates $f_{n}(j)-t h$ row. Here, $f_{n}(j)$ is a function that maps $\{2,3\}$ to $\{1,2,3\}$ with the property that $1 \leq f_{n}(j) \leq j$. Let $\delta_{s, t}=\left\{\begin{array}{ll}1, & s=t \\ 0, & s \neq t\end{array}\right.$. Then there is a permutation $\pi_{n}$ such that

$$
K_{n}=P_{\pi_{n}}\left(\begin{array}{ccc}
k_{1}^{(n)} & \delta_{1, f_{n}(2)} k_{2}^{(n)} & \delta_{1, f_{n}(3)} k_{3}^{(n)} \\
0 & \delta_{2, f_{n}(2)} k_{2}^{(n)} & \delta_{2, f_{n}(3)} k_{3}^{(n)} \\
0 & 0 & \delta_{3, f_{n}(3)} k_{3}^{(n)}
\end{array}\right) .
$$


From $\sum_{n} K_{n}^{\dagger} K_{n}=I$, we get that

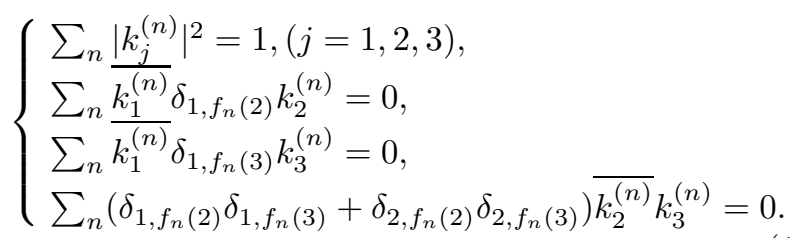

For $|\psi\rangle=\left(\psi_{1}, \psi_{2}, \psi_{3}\right)^{t}$, by a direct computation, one can get

$K_{n}|\psi\rangle=P_{\pi_{n}}\left(\begin{array}{c}k_{1}^{(n)} \psi_{1}+\delta_{1, f_{n}(2)} k_{2}^{(n)} \psi_{2}+\delta_{1, f_{n}(3)} k_{3}^{(n)} \psi_{3} \\ \delta_{2, f_{n}(2)} k_{2}^{(n)} \psi_{2}+\delta_{2, f_{n}(3)} k_{3}^{(n)} \psi_{3} \\ \delta_{3, f_{n}(3)} k_{3}^{(n)} \psi_{3}\end{array}\right)$

and so

$$
\left\{\begin{array}{l}
k_{1}^{(n)} \psi_{1}+\delta_{1, f_{n}(2)} k_{2}^{(n)} \psi_{2}+\delta_{1, f_{n}(3)} k_{3}^{(n)} \psi_{3}=\alpha_{n} \phi_{\pi_{n}^{-1}(1)}, \\
\delta_{2, f_{n}(2)} k_{2}^{(n)} \psi_{2}+\delta_{2, f_{n}(3)} k_{3}^{(n)} \psi_{3}=\alpha_{n} \phi_{\pi_{n}^{-1}(2)}, \\
\delta_{3, f_{n}(3)} k_{3}^{(n)} \psi_{3}=\alpha_{n} \phi_{\pi_{n}^{-1}(3)} .
\end{array}\right.
$$

Applying $\sum_{n}|\cdot|^{2}$ to above equations, we have

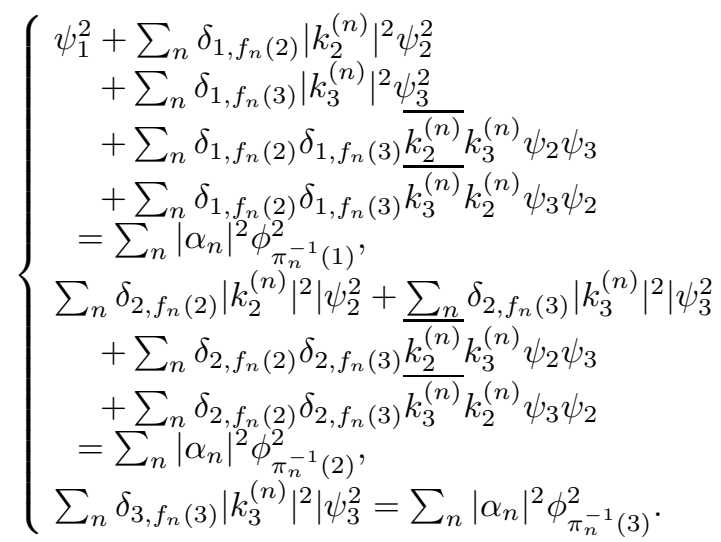

Note that, for $s=1,2,3$,

$$
\begin{aligned}
& \sum_{n}\left|\alpha_{n}\right|^{2} \phi_{\pi_{n}^{-1}(s)}^{2} \\
= & \sum_{n, \pi_{n}^{-1}(s)=1}\left|\alpha_{n}\right|^{2} \phi_{1}^{2}+\sum_{n, \pi_{n}^{-1}(s)=2}\left|\alpha_{n}\right|^{2} \phi_{2}^{2} \\
& +\sum_{n, \pi_{n}^{-1}(s)=3}\left|\alpha_{n}\right|^{2} \phi_{3}^{3},
\end{aligned}
$$

Let $d_{i j}=\sum_{n, \pi_{n}^{-1}(i)=j}\left|\alpha_{n}\right|^{2}, 1 \leq i, j \leq 3$, then the matrix $D=\left(d_{i j}\right)$ is a doubly stochastic matrix, since $\sum_{n}\left|\alpha_{n}\right|^{2}=$ 1. Furthermore,

$$
\begin{aligned}
& D\left(\phi_{1}^{2}, \phi_{2}^{2}, \phi_{3}^{2}\right)^{t} \\
= & \left(\sum_{n}\left|\alpha_{n}\right|^{2} \phi_{\pi_{n}^{-1}(1)}^{2}, \sum_{n}\left|\alpha_{n}\right|^{2} \phi_{\pi_{n}^{-1}(2)}^{2}, \sum_{n}\left|\alpha_{n}\right|^{2} \phi_{\pi_{n}^{-1}(3)}^{2}\right)^{t} .
\end{aligned}
$$

This implies that

$$
\begin{aligned}
& \left(\sum_{n}\left|\alpha_{n}\right|^{2} \phi_{\pi_{n}^{-1}(1)}^{2}, \sum_{n}\left|\alpha_{n}\right|^{2} \phi_{\pi_{n}^{-1}(2)}^{2}, \sum_{n}\left|\alpha_{n}\right|^{2} \phi_{\pi_{n}^{-1}(3)}^{2}\right)^{t} \\
\prec & \left(\phi_{1}^{2}, \phi_{2}^{2}, \phi_{3}^{2}\right)^{t} .
\end{aligned}
$$

On the other hand, note that in equation (21),

$$
\begin{aligned}
& \sum_{n} \delta_{1, f_{n}(2)}\left|k_{2}^{(n)}\right|^{2} \psi_{2}^{2} \\
& \quad+\sum_{n} \delta_{1, f_{n}(3)}\left|k_{3}^{(n)}\right|^{2} \underline{\psi_{3}^{2}} \\
& \quad+\sum_{n} \delta_{1, f_{n}(2)} \delta_{1, f_{n}(3)} \overline{k_{2}^{(n)}} k_{3}^{(n)} \psi_{2} \psi_{3} \\
& \quad+\sum_{n} \delta_{1, f_{n}(2)} \delta_{1, f_{n}(3)} k_{3}^{(n)} k_{2}^{(n)} \psi_{3} \psi_{2} \\
& =\sum_{n}\left|\delta_{1, f_{n}(2)} k_{2}^{(n)} \psi_{2}+\delta_{1, f_{n}(3)} k_{3}^{(n)} \psi_{3}\right|^{2} .
\end{aligned}
$$

From the definition of majorization and equations (18),(21), one can check that

$$
\prec\left(\sum_{n}^{2}\left|\alpha_{n}\right|^{2} \phi_{\pi_{n}^{-1}(1)}^{2}, \sum_{n}\left|\alpha_{n}\right|^{2} \phi_{\pi_{n}^{-1}(2)}^{2}, \sum_{n}\left|\alpha_{n}\right|^{2} \phi_{\pi_{n}^{-1}(3)}^{2}\right)^{t} .
$$

Therefore $\left(\psi_{1}^{2}, \psi_{2}^{2}, \psi_{3}^{2}\right)^{t} \prec\left(\phi_{1}^{2}, \phi_{2}^{2}, \phi_{3}^{2}\right)^{t}$.

Outlook. - Our results raise many interesting questions. It would be of great interest to determine when a mixed state $\rho$ can be transformed to a mixed state $\sigma$ by incoherent operations. What we get is if $\sigma$ is incoherent then there exists an incoherent operation $\Phi$ such that $\Phi(\rho)=\sigma$ for any state $\rho$. We show this by explicitly constructing an incoherent operation that achieves the transformation in the Appendix. What are sufficient conditions for the existence of catalysts? Finally, all of considerations above implicitly assumed the finite dimensional setting, but this is neither necessary nor desirable as there are very relevant physical situations that require infinite dimensional systems for their description. Most notable are the quantum states of light, that is quantum optics, with its bosonic character requires infinite dimensional systems, harmonic oscillators, for their description. Hence, coherence manipulation and existence of catalysts in infinite dimensional systems are needed. Mirroring analogous developments in entanglement manipulation [22], we expect that the manipulation of coherence in infinite dimensional systems can be built.

Conclusions. - In this manuscript, we give a complete characterization of coherence manipulation for pure states in terms of majorization. This result offers an affirmative answer to the open question: whether, given two states $\rho$ and $\sigma$, either $\rho$ can be transformed into $\sigma$ or vice versa under incoherent operations [13]. The proof of the result also provides a effective constructive method to find the incoherent operation transforming $|\psi\rangle$ to $|\phi\rangle$, whenever $\left(\left|\psi_{1}\right|^{2}, \cdots,\left|\psi_{d}\right|^{2}\right)^{t} \prec\left(\left|\phi_{1}\right|^{2}, \cdots,\left|\phi_{d}\right|^{2}\right)^{t}$. The majorization approach used here is similar to that used to establish the ordering of entanglement states, which led to advancement in the field of quantum computation. Based on Theorem 1, some interesting properties of coherent catalysts are discovered.

Acknowledgement. - The authors thank referees for their valuable comments which improve the presentation of this paper. This work was completed while the authors were visiting the Institute of Quantum Computation of the University of Waterloo during the academic year 2014-2015 under the support of China Scholarship Council. We thank Professor David W. Kribs and Professor Bei Zeng for their hospitality. This work is partially 
supported by the Natural Science Foundation of China (No. 11001230, 11301312, 11171249), the Natural Science Foundation of Fujian (2013J01022, 2014J01024), the Natural Science Foundation of Shanxi ( No. 2013021001-
1, 2012011001-2) and the Research start-up fund for Doctors of Shanxi Datong University under Grant No. 2011B-01.
[1] M. A. Nielsen and I. L. Chuang, Quantum Computation and Quantum information (Cambridge University Press, Cambridge, 2000).

[2] C.H. Bennett et al., Phys. Rev. A 54, 3824 (1996).

[3] C.H. Bennett et al., Phys. Rev. A 53, 2046 (1996).

[4] C.H. Bennett et al., Phys. Rev. Lett. 76, 722 (1996).

[5] V. Vedral et al., Phys. Rev. Lett. 78, 2275 (1997).

[6] V. Vedral and M.B. Plenio., Phys. Rev. A 57, 1619 (1998).

[7] M.A. Nielsen, Phys. Rev. Lett. 83, 436 (1999).

[8] G. Vidal, Phys. Rev. Lett. 83, 1046 (1999).

[9] D. Jonathan and M.B. Plenio, Phys. Rev. Lett. 83, 1455 (1999).

[10] D. Jonathan and M.B. Plenio, Phys. Rev. Lett. 83, 3566 (1999).

[11] L. Hardy, Phys. Rev. A 60, 1912 (1999).

[12] M.A. Nielsen, G. Vidal, Quantum Inf. Comput. 1, 76 (2001).

[13] T. Baumgratz, M. Cramer, M.B. Plenio, Phys. Rev. Lett. 113, 140401 (2014).

[14] R. Bhatia, Matrix Analysis (Springer-Verlag, New York, 1997).

[15] I. Ghiu, M. Bourennane, A. Karlsson, Phys. Lett. A 287, 12 (2001).

[16] W. Song, Y. Huang, N.L. Liu, Z.B. Chen, J. Phys. A: Math. Gen. 40, 785 (2007).

[17] I. Chattopadhyay, D. Sarkar, Phys. Rev. A 77, (2008) 05305(R).

[18] G. Bowen, N. Datta, IEEE Trans. Inf. Theory 54, 3677 (2008).

[19] J. Åberg, Phys. Rev. Lett. 113, 150402 (2014).

[20] D. Girolami, Phys. Rev. Lett. 113, 170401 (2014).

[21] S.-P. Du, Z.-F. Bai, X.-F. Qi, arXiv:1504.02862v1.

[22] M. Owari, S.L. Braunstein, K. Nemoto, M. Murao, Quantum Inf. Comput. 8, 30 (2008).

\section{Appendix : Transition of mixed states}

We will show if the output mixed state $\sigma$ is incoherent, i.e., $\sigma \in \mathcal{I}$, then for any quantum state $\rho$, there exists an incoherent operation $\Phi$ such that $\Phi(\rho)=\sigma$. We do this by an explicit construction of an incoherent operation. Define an incoherent operation

$$
\Phi_{1}(\rho):=\sum_{i=1}^{d}|i\rangle\langle i|\rho| i\rangle\langle i| .
$$

The effect of this operation is to remove all off-diagonal elements of $\lambda_{i, j}|i\rangle\langle j|(i \neq j)$ from $\rho=\sum_{i, j=1}^{d} \lambda_{i, j}|i\rangle\langle j|$, leaving the diagonal elements $\lambda_{i, i}|i\rangle\langle i|$ intact. Denote $\left\{\lambda_{i}=\langle i|\rho| i\rangle\right\}_{i=1}^{d}$ and $\left\{\mu_{i}\right\}_{i=1}^{d}$ the eigenvalues of $\Phi_{1}(\rho)$ and $\sigma$, respectively. Let

$$
\begin{aligned}
A_{1}= & \sqrt{\mu_{1}}|1\rangle\left\langle 1\left|+\sqrt{\mu_{2}}\right| 2\right\rangle\left\langle 2\left|+\cdots+\sqrt{\mu_{d}}\right| d\right\rangle\langle d|, \\
A_{2}= & \sqrt{\mu_{2}}|1\rangle\left\langle 2\left|+\sqrt{\mu_{3}}\right| 2\right\rangle\langle 3|+\cdots \\
& +\sqrt{\mu_{d}}|d-1\rangle\left\langle d\left|+\sqrt{\mu_{1}}\right| d\right\rangle\langle 1|, \\
\cdots, & \\
A_{i}= & \sqrt{\mu_{i}}|1\rangle\left\langle i\left|+\cdots+\sqrt{\mu_{m_{s+i-1}}}\right| s\right\rangle\left\langle m_{s+i-1}\right|+\cdots \\
& +\sqrt{\mu_{m_{d+i-1}}}|d\rangle\left\langle\mu_{m_{d+i-1}}\right|, \\
\cdots, & \\
A_{d}= & \sqrt{\mu_{d}}|1\rangle\left\langle d\left|+\sqrt{\mu_{1}}\right| 2\right\rangle\left\langle 1\left|+\cdots+\sqrt{\mu_{d-1}}\right| d\right\rangle\langle d-1|,
\end{aligned}
$$

here $m_{x}=x-\left[\frac{x-1}{d}\right] d$. It is easy to check that $\sum_{i=1}^{d} A_{i} A_{i}^{\dagger}=I$. By a direct computation, one can get $\Phi_{2}\left(\Phi_{1}(\rho)\right)=\sum_{i=1}^{d} A_{i}^{\dagger} \Phi_{1}(\rho) A_{i}=\sigma$. Let $\Phi=\Phi_{2} \circ \Phi_{1}$, then $\Phi$ is an incoherent operation satisfying $\Phi(\rho)=\sigma$. 